\title{
Estimativa da ingestão de ferro e vitamina C em adolescentes no ciclo menstrual
}

\author{
Estimate of iron and vitamin C intake \\ in adolescents during the menstrual cycle phases
}

Luciana Azevedo ${ }^{1}$

Hércia Stampini Duarte M artino ${ }^{2}$

Flávia Giolo Carvalho ${ }^{1}$

M arcelo Lacerda Rezende ${ }^{1}$

\footnotetext{
${ }^{1}$ Setor de N utrição, Departamento deCiências Biológicas, Universidade Federal deAlfenas. Rua Gabriel M onteiro da Silva 714/sala H 206, Centro. 37130-000 Alfenas MG. lazevedo@unifal-mg.edu.br ${ }^{2}$ Universidade Federal de Viçosa.
}

Abstract The vitamin $C$ and iron ingestion level was evaluated in adolescents during thethreephases of the menstrual cycle. The food intake of 30 adolescents was analyzed during the premenstrual, menstrual, and postmenstrual phases, by the registration technique, with the analysis of two registrations per individual, in each phase. The prevalence estimate was done considering six dietary registrations for each adolescent. The patterns used as reference were the Dietary Reference Intakes (DRI) - Estimated Average Requirement (EAR) from Institute of M edicine - IOM (2001), using the probability approaches and the cut point method for the iron and vitamin $C$ respectively, in addition to the values from the Recommended Dietary Allowances ( $N$ ational Research Council). The adolescents were aged $15.5 \pm 1.7$; the age of the menarche was $11.8 \pm 1,1$; and gynecological age $3.6 \pm 1.9$. The medial consumption of iron was $10.6 \mathrm{mg} /$ day and of vitamin C $77.6 \mathrm{mg} /$ day. It was observed by DRIs-EAR an estimate of inadequacy for iron of $22.6 \%$ and for vitamin $\mathrm{C}$ of $34 \%$ and by National Research Council, $63.3 \%$ and $20 \%$, respectively. Despite the divergence among the patterns, both results shows a nutritional risk for the studied population. The accomplishment of wider dietary studies, using D RI-EAR is suggested, favoring nutritional diagnoses with subsequent evaluation of intervention measures. Key words Adolescence, Iron, M enstrual cycle, Eating
Resumo Avaliou-se o nível de ingestão deferro e vitamina $C$, em adolescente nas três fases do ciclo menstrual. 0 consumo alimentar de trinta adolescentes foi analisado pela técnica de registro, durante as fases pré-menstrual, menstrual e pósmenstrual, com a análise de dois registros por indivíduo, em cada fase. A prevalência da ingestão foi feita considerando seis registros alimentares de cada adolescente. Os padrões utilizados como referência foram as Dietary Reference Intakes (DRI)- Estimated Average Requirement (EAR) do Institute of M edicine (IOM), utilizando as abordagens probabilística e ponto de corte, para 0 ferro e vitamina $C$, respectivamente, além dos valores do Recommended Dietary Allowances (National Research Council). As adolescentes tinham 15,5 $\pm 1,7$ anos, com idade da menarca de $11,8 \pm 1,1$ anos eginecológica de $3,6 \pm 1,9$ anos. 0 consumo mediano de ferro foi $10,6 \mathrm{mg} /$ dia e de vitamina C, 77,6 mg/dia. Observou-se pelas D RIEAR uma prevalência de inadequação para ferro de $22,6 \%$ e de vitamina C $34 \%$ e pelo National Research Council, de 63,3\% e 20\%, respectivamente. A pesar da divergência entre os padrões, os resultados apontam risco nutricional para a população estudada. Sugere se a realização de mais estudos di etéticos, utilizando as DRI-EAR, favorecendo diagnósti cos nutricionais com subsequente medidas de intervenção.

Palavras-chave Adolescência, Ingestão alimentar, Vitamina C, Ferro, Ciclo menstrual 
Introdução

A adolescência é uma etapa evolutiva peculiar ao ser humano, que culmina todo o processo maturativo biopsicossocial do indivíduo. Caracteriza-se por profundas transformações somáticas, psicológicas e sociais, compreendendo, de acordo com a O rganização M undial deSaúde, as idades de dez a dezenove anos ${ }^{1}$. Nessa fase de crescimento acelerado, é de grande importância a atenção a alguns nutrientes, como o ferro, 0 cálcio e as vitaminas A e C, cujas necessidades, aumentadas, estão fortemente ligadas ao padrão decrescimento ${ }^{2}$.

A avaliação do consumo alimentar tem sido uma área de grande interesse, especialmente quando associado às situações específicas, como a adolescência e o ciclo menstrual, que envolvem modificações sistemáticas na ingestão qualitativa e quantitativa de macro e micronutrientes ${ }^{3,4}$.

A adolescência corresponde a um estágio de mudança de comportamento de saúde. Vários estudos têm mostrado alterações comportamentais durante esse período, associadas à al imentação, como hábitos de sefazer dieta eomitir refeições ${ }^{5,6}$. Torna-se evidente a falta de adequação nutricional, em decorrência da utilização de die tas para 0 alcance da imagem corporal esquálida, idealizada como padrão dietético ${ }^{7}$. Assim, as alterações repercutem na variação do conteúdo calórico, de macro e micronutrientes, com consumo de dietas caracterizadamente hipoglicídicas, hiperprotéicas, hiperlipídicas, deficientes em ferro, vitamina A ecálcio. Em decorrência do crescimento rápido, do aumento da massa muscular, do volume sanguíneo e das enzimas respiratórias, que ocorrem durante a adolescência, a variação da necessidade de ferro é aumentada para ambos os sexos 8 .

Associadas a essas mudanças de comportamento, implícitas da adolescência, também são acrescidas aquelas referentes às diferentes fases do ciclo menstrual, as quais interferem na seleção ou ingestão de alimentos ${ }^{8}$. Além disso, o sexo feminino possui um aumento adicional da necessidade de ferro, com o advento da menarca, devido às perdas durante a menstruação ${ }^{9}$. Esses fatores tornam esse grupo vulnerável a deficiências desse mineral, incluindo a sua forma mais severa, a anemia ${ }^{10}$.

A anemia é a carência nutricional mais comum no mundo e com maior prevalência em grupos populacionais mais vulneráveis ${ }^{10}$, sendo reconhecida como principal problema nutricio- nal dos adolescentes. De 39 estudos revisados ${ }^{1}$, quanto à prevalência deanemia em adolescentes, 32 ocorreram em países em desenvolvimento. A pesar desses dados, as estatísticas do status nutricional de mulheres em países em desenvolvimento, mesmo desconsiderando o estágio de vida, são menos disponíveis e incompletas, com dados ainda concentrados em pré-escolares ena população como um todo. Esse desconhecimento tem impacto negativo, prejudicando o desenvolvimento de políticas de intervenção eprevenção, contribuindo para a manutenção desse panorama e suas consequências, como número de óbitos durante 0 parto ${ }^{3,11}$.

$N$ ão é somente a baixa ingestão de ferro que acarreta elevada prevalência de anemia. Outros fatores, como baixa ingestão de vitamina $\mathrm{C}$, em referência ao ácido ascórbico e deidroascórbico, dietas inadequadas para perda de peso e adoção de dietas vegetarianas desajustadas são associadas aos riscos aumentados de anemia ${ }^{1}$.

$\mathrm{Na}$ prevenção da anemia ferropriva, a vitamina $C$ possui efeito benéfico, agindo como agente facilitador da absorção do ferro não-heme ${ }^{12}$. Vitolo e Bortolini ${ }^{13}$ observaram que crianças não anêmicas consumiram significativamente mais vitamina $C$ do que as anêmicas, ratificando que a composição da dieta, e não a quantidade total dealimentos, éo elemento de maior importância na prevenção da anemia ferropriva. Esse aspecto toma relevância em estratos populacionais em queo consumo de carneélimitado em função de condições econômicas. Contrariamente, Reddy, H urrell e Cook ${ }^{14}$ não observaram relação significante entre a absorção de ferro, heme ou não, e diferentes níveis de vitamina $C$, cálcio, fósforo, fibra. Com relação ao consumo de vitamina $\mathrm{C}$, a sua necessidade é de fácil alcance pelo consumo de frutas, vegetais e sucos, além de sua possível suplementação $0^{15}$.

0 desequilíbrio na ingestão demicronutrientes torna essa fase ainda mais vulnerável, influenciando de forma desfavorável o desenvolvimento pubertário eo crescimento somático ${ }^{1,16}, 0$ quejustifica levantamentos e intervenções nutricionais nesse estágio de vida. Também acresce 0 fato de que o melhor entendimento da ingestão de alimentos durante as fases do ciclo menstrual pode, ainda, contribuir para o tratamento dasíndrome pré menstrual, desordens alimentares e obesidade 8 . Dessa forma, no presente trabalho, objetivou-se avaliar o nível de ingestão deferro e vitamina $\mathrm{C}$ em adolescentes nas três fases do ciclo menstrual. 
M etodologia

\section{Obtenção dos dados}

$\mathrm{Na}$ realização deste estudo, foi utilizado o banco de dados de Azevedo ${ }^{17}$, referenteà pesquisa conduzida nos municípios de Alfenas e Areado (M G). Para a coleta desses dados, constituiuse uma amostra inicial composta por sessenta adolescentes do sexo feminino, com idade variando entre catorze e dezoito anos, não usuárias decontraceptivos ou medicamentos de quaisquer espécies, que relataram apresentar bom estado de saúde durante todo o período experimental e ausência de patologia aguda ou crônica clinicamente evidente. Essas exigências estão de acordo com as propostas por estudos semelhantes ${ }^{8,18}$.

Preconizou-sea sel eção de idadeginecológica maior ou igual a doze meses ${ }^{19}$. 0 recrutamento das adolescentes foi realizado de forma aleatória, em oito escolas públicas e privadas situadas em pontos equidistantes nos municípios citados. A pesquisa atendeu às normas regulamentares depesquisa envolven do seres humanos efoi aprovada pelo comitê de ética em pesquisa da Universidade Federal de Alfenas.

0 método utilizado para a obtenção dos dados referentes ao consumo alimentar das adolescentes durante o ciclo menstrual foi o registro alimentar ${ }^{20-22}$. Para essa finalidade, o levantamento ocorreu duranteas fases pré-menstrual, menstrual e pós-menstrual, com a análise de dois re gistros de cada indivíduo, em cada fase, totalizando seis registros para cada adolescente. I nicialmente, assumiu-se o ciclo menstrual de 28 dias e as variações individuais foram consideradas durante o levantamento do calendário menstrual para cada participante. 0 período pré menstrual (fase luteínica) foi considerado aquele precedente ao sangramento, sete a três dias antes; 0 menstrual ocorreu durante a fase de sangramento, do segundo ao quinto dia após seu início, desdeque esteainda semantivesse; e o pós-menstrual (fase folicular), do décimo ao décimo quarto dia após o início do sangramento ${ }^{23-25}$.

$\mathrm{O}$ estudo foi dividido em três etapas. Na primeira, as adolescentes foram submetidas a orientações sobre a pesquisa, à assinatura do termo de consentimento, cadastramento, ficha de anamnese, calendário menstrual e a idade ginecológica, obtida pela diferença entre a data da menarca e 0 dia de aplicação do questionário ${ }^{26}$.

$\mathrm{Na}$ segunda fase, elas foram orientadas e assistidas durante o preenchimento do formulário de registro dietético de 24 horas, no qual deveri- am ser rigorosamente consideradas todas as refeições diárias, como desjejum, lanche, almoço, jantar e ceia; horário das refeições; caracterização dos alimentos consumidos; formas de preparo; marcas comerciais, em função da elevada frequência de produtos enriquecidos com micronutrientes, eas quantidades em medidas casei ras e medidas de volume. Esses aspectos permitiram uma análise qual itativa equantitativa da dieta ${ }^{13,27}$. Para referência demedidas, foi utilizado o álbum fotográfico ${ }^{28}$, com o objetivo de auxiliar a adolescente na estimativa da quantidade de alimentos consumidos.

A terceira etapa constava do preenchimento de dois formulários, durante as três fases do ciclo menstrual, o que ocorreria durante o período de dois meses. Essa teve como objetivo assegurar 0 cumprimento do delineamento experimental, devido às irregularidades menstruais comumente observadas em adolescentes ${ }^{19}$, e permitir a estimativa da variabilidade do consumo diário de nutrientes, por obter duas medidas independentes ${ }^{21}$.

Ao final, do total de sessenta adolescentes, somente trinta permaneceram na pesquisa. Foram utilizados como critérios de suspensão o não cumprimento do calendário experimental para reuniões de treinamento e a entrega dos registros alimentares einconsistência no seu preenchimento ${ }^{13}$. 0 tamanho final da amostra, de trinta adolescentes, está de acordo com Slater et al. ${ }^{21}$, Fisberg et al. ${ }^{29}$ e Institute of $M$ edicine ${ }^{20}$, para avaliações deestimativa deprevalência de inadequação.

Para o cálculo nutricional das dietas, foi utilizado o programa Diet Pro $4^{30}$. Para alimentos que não constavam no banco de dados do programa, foram utilizadas tabelas de composição química dos alimentos ${ }^{31-33}$, além dos rótulos de produtos industrializados não referidos nas tabelas ${ }^{13}$. No caso dos alimentos e/ou preparações possuírem marcas comerciais diferentes, optouse por aqueles com maior número de informações quanto aos aspectos nutricionais ${ }^{34}$.

\section{Avaliação do consumo de nutrientes em grupos de adolescentes}

Para o cálculo da estimativa da prevalência de inadequação da ingestão de ferro e vitamina C da população de adolescentes, foram utilizados como padrões de referência as Dietary Referencelntakes- Estimated Average Requirement ${ }^{20,35}$.

Inicialmente, testou-sea existência dediferenças significativas no consumo de ferro evitamina $\mathrm{C}$ durante os três períodos, pré menstrual, menstrual e pós-menstrual. Para isso, foi utilizado 0 
teste não paramétrico de Kruskal-Wallis. Nesse teste, todos os dados são ordenados numericamente e os valores da ordenação são somados em cada grupo a ser comparado. 0 teste de Kruskal-Wallis procura determinar se a mediana da ordenação diferemais do que se esperaria apenas por acaso ${ }^{36}$. Como os resultados dessa comparação (Kruskal-Wallis, $p<0,05$ ) demonstraram que os consumos, tanto do ferro quanto da vitamina $C$, foram semelhantes durante as três fases do ciclo menstrual, a estimativa de inadequação foi calculada considerando os seis registros alimentares de cada adolescente. A partir de então, os cálculos da inadequação do ferro e da vitamina $C$ seguiram métodos diferentes.

Para a estimativa da inadequação do ferro, foi utilizada a abordagem probabilística manualmente determinada, conformeestabelecido por $10 \mathrm{M}^{20}$. Essa abordagem é necessária, uma vez que a curva de distribuição das necessidades de ferro é considerada assimétrica, devido às maiores necessidades desse mineral pelas mulheres pós-menarca. Diferentemente, para a vitamina $C$, em que a pressuposição da si metria na distribuiçãa das necessidades é satisfeita, foi utilizada a abordagem do ponto de corte, conforme estabelecido em Fisberg et al. ${ }^{29} \mathrm{e}$ Slater et al. ${ }^{21}$.
0 modelo proposto para a análise da inadequação do ferro é de fácil execução e os passos estão apresentados a seguir, tomando-se como base a Tabela 1, apresentada nos resultados. A primeira e a segunda coluna desta tabela foram obtidas do IOM ${ }^{20}$. N ela, observa-se que as ingestões abaixo de 4,6 mg/dia de ferro são assumidas ter $100 \%$ de probabilidade de inadequação ( risco $=1,0$ ), enquanto as ingestões acima de $14,8 \mathrm{mg} /$ dia são assumidas ter risco zero de inadequação. Para ingestões entreesses extremos, cada intervalo apresentado na segunda coluna da Tabela 1 possui uma probabilidade de inadequação. 0 risco apropriado de inadequação corresponde ao número demulheres com ingestões nessa faixa multiplicado pela probabilidade de inadequação. Por exemplo, obtiveram-se duas adolescentes com ingestão entre 6,3 a 6,9 mg/dia, então o número de indivíduos com ingestão inadequada foi de 1,5 $(2 \times 0,75)$. Esse cálculo foi continuado para cada faixa de ingestão, obtendo-se ao final o total de 6,8 adolescentes com ingestão inadequada, o que corresponde a $22,6 \%$ da população pesquisada.

A estimativa da inadequação do consumo da vitamina $\mathrm{C}$ foi feita de acordo com o descrito por Fisberg et al..$^{22}$ eSlater et al. ${ }^{21}$. I nicial mente, a normalidade dos dados foi testada, utilizando-

Tabela 1. Abordagem probabilística para estimar a prevalência de inadequação do ferro em um grupo de 30 adolescentes, durante as três fases do ciclo menstrual.

\begin{tabular}{lccc}
\hline $\begin{array}{c}\text { Probabilidade } \\
\text { de inadequação }\end{array}$ & $\begin{array}{c}\text { Intervalos de consumos } \\
\text { usuais (mg/d) }\end{array}$ & $\begin{array}{c}\text { Número de } \\
\text { adolescentes }\end{array}$ & $\begin{array}{c}\text { Número de adolescentes } \\
\text { com ingestão inadequada }\end{array}$ \\
\hline 1 & $<4,63$ & 0 & 0 \\
0,96 & 4,64 a 5,06 & 0 & 0 \\
0,93 & 5,07 a 5,61 & 0 & 0 \\
0,85 & 5,62 a 6,31 & 0 & 0 \\
0,75 & 6,32 a 6,87 & 2 & 1,5 \\
0,65 & 6,88 a 7,39 & 1 & 0,65 \\
0,55 & 7,40 a 7,91 & 1 & 0,55 \\
0,45 & 7,92 a 8,48 & 2 & 0,9 \\
0,35 & 8,49 a 9,15 & 4 & 1,4 \\
0,25 & 9,16 a 10,03 & 1 & 0,25 \\
0,15 & 10,04 a 11,54 & 8 & 1,2 \\
0,08 & 11,55 a 13,08 & 0 & 0 \\
0,04 & 13,09 a 14,80 & 8 & 0,32 \\
0 & $>14,80$ & 3 & 0 \\
Total de adolescentes & & 30 & 6,77 \\
Estimativa de inadequação $(\%)$ & & & 22,6 \\
\hline
\end{tabular}

(a) Fonte: $10 M^{20}$.

(b) Interval os de consumos usuais em adolescentes do sexo feminino de 14 a 18 anos, não usuárias de contraceptivos orais (mg/d), fornecidos pelo IOM. Fonte: IOM ${ }^{20}$. 
se 0 teste de Kolmogorov-Smirnov. Como os dados não apresentavam uma distribuição normal, os mesmos foram logaritimizados para o alcance da normalidade e novamente testados quanto a sua distribuição, quando a atenderam. Após essa transformação, foi feita a análise de variância e o cálculo da razão entre a variabilidade interpessoal e intrapessoal. A seguir foi calculada a prevalência de inadequação.

Por fim, também calculou-se a inadequação do consumo desses nutrientes, utilizando-se valores dereferência entre 80 a $120 \%$ do preconizado pelo National Research Council ${ }^{37}$, conforme outros autores $18,34,38$.

\section{Resultados}

A amostra desse estudo compreendeu 30 adolescentes com idade média de 15,5 $\pm 1,7$ anos. Quanto à anamnese referente ao ciclo menstrual, as adolescentes apresentaram a média de idade da menarca de $11,8 \pm 1,1$ anos e idade ginecológica de $3,6 \pm 1,9$.
Com relação ao consumo dos nutrientes, a Tabela 1 apresenta a abordagem probabilística para estimativa da prevalência da inadequação do ferro. A Tabela 2 apresenta os resultados referentes ao consumo em cada fase do ciclo menstrual, observando-se que não houve diferença estatística entre eles, permitindo que a porcentagem de inadequação da vitamina $C$ e do Ferro fosse obtida dos valores referentes a seis repetições. Observa-se que quanto ao ferro, 6,8 muIheres tiveram ingestões inadequadas, determinando uma estimativa de prevalência de inadequação de $22,6 \%$ ea vitamina C apresentou $34 \%$ de inadequação (Tabela 2). Quando foi utilizado a avaliação pelo National Research Council, encontrou-se uma inadequação de 19 (63,3\%) e 6 (20\%), referentes ao ferro e vitamina $C$, respectivamente (Quadro 1).

\section{Discussão}

Os aspectos nutricionais relacionados ao ciclo menstrual têm sido associados aos fatores en-

Tabela 2. M ediana do consumo de ferro e vitamina C e probabilidade de inadequação entre as fases do ciclo menstrual das adolescentes.

\begin{tabular}{|c|c|c|c|c|c|c|}
\hline Nutriente & $\begin{array}{l}\text { Pré-menstrual } \\
\qquad(\mathrm{mg} / \mathrm{dia})\end{array}$ & $\begin{array}{l}\text { Menstrual } \\
\text { (mg/dia) }\end{array}$ & $\begin{array}{l}\text { Pós-menstrual } \\
\text { (mg/dia) }\end{array}$ & $\begin{array}{l}\text { M ediana do consumo } \\
\text { total (mg/dia) }\end{array}$ & $\begin{array}{c}\text { EAR } \\
\text { (mg/dia) }\end{array}$ & $\begin{array}{l}\text { \% inadequação } \\
(E A R)\end{array}$ \\
\hline Ferro & 10,4 & 10,7 & 10,7 & 10,6 & $7,9 *$ & 22,6 \\
\hline Vitamina C & 58,8 & 82,0 & 78,6 & 77,6 & $56 *$ & 34,0 \\
\hline
\end{tabular}

* Para mulheres no estágio de vida de 14 a 18 anos deidade.

**Comparação ao National Research Council ${ }^{37}$, conformeU rbano et al. ${ }^{18}$.

Quadro 1. Adequação do consumo alimentar das adolescentes utilizando como padrão a recomendação do National Research Council ${ }^{37}$.

\begin{tabular}{|l|r|c|c|c|}
\hline \multicolumn{1}{|c|}{ Nutriente } & Adequado & Insuficiente & Elevado & \% inadequação** \\
\hline Ferro & & & & \\
$\quad$ Padrão de referência (mg/dia)* & $12-18$ & $<12$ & $>18$ & \\
$\quad$ Número de adolescentes & 10 & 19 & 1 & 63,3 \\
\hline Vitamina C & & & & \\
$\quad$ Padrão de referência (mg/dia) & $48-72$ & $<48$ & $>72$ & 20,0 \\
Número de adolescentes & 5 & 6 & 19 & \\
\hline
\end{tabular}

*Para mulheres no estágio de vida de 14 a 18 anos de idade.

** comparação ao National Research Council ${ }^{37}$, conforme U rbano et al. ${ }^{18}$. 
volvidos na fisiologia da menstruação, determinam um ciclo menstrual com duração média de 28 dias, podendo variar de 20 a $45^{39}$, ou de 21 a 40 dias com duração do sangramento de dois a oito dias na adolescência ${ }^{40}$, os quais são concordantes com os encontrados no presente estudo. 0 desenvolvimento biológico, avaliado pela idade da menarca, foi semelhante ao encontrado no Brasil por M antoanelli, VitaleeAmânci ${ }^{41}$, onde a média de idade da menarca foi de 12,2 anos.

Apesar de inclusão somente de adolescentes com idade ginecológica maior ou igual a doze meses, com a finalidade de excluir aquelas com maior probabilidade de estarem no período mais intenso de mudanças físicas, relacionadas à puberdade ${ }^{19}, 70 \%$ delas ainda relataram ter ciclo menstrual irregular. Essa irregularidade influenciou no desenvolvimento do estudo, acarretando perda dos registros, principalmente aqueles da fase pré menstrual, os quais eram tomados em ciclos consecutivos. De acordo com Tarasuk e Beaton ${ }^{8}$, considerar os catorze dias da fase pré menstrual como a fase lútea é uma prática comum e variações individuais no tamanho do ciclo menstrual geralmente ocorrem em decorrência da fase folicular, pós-menstrual.

Com relação à ingestão de alimentos, o ciclo menstrual não influencia apenas 0 apetite e 0 tamanho das refeições. A literatura também relata mudanças em tipos de macronutrientes ingeridos, além de citar alterações na seleção de produtos alimentícios, bem como desenvolvimento decompulsão por determinados alimentos ${ }^{24}$. Entretanto, ao investigar a ingestão de ferro e de vitamina C, não foi encontrada diferença significativa entre as três fases do ciclo menstrual, determinando que a estimativa da prevalência de inadequação da ingestão desses nutrientes fosse realizada com o consumo médio das três fases e, subsequentemente, comparada a padrões de referência, que foram as Dietary Reference Intakes (DRIS), com a utilização das tabelas de EAR (Estimated Average Requirement) eo National Research Counci ${ }^{21,37,42,43}$

Para a estimativa de prevalência deinadequação, a literatura apresenta como métodos estatísticos a "abordagem probabilística" e a "EAR como "ponto de corte". A prevalência de inade quação é a proporção de indivíduos cujo consumo está abaixo da EAR ${ }^{21,35,44}$.

A EAR como ponto de corte pode ser utilizada desde que obedecidas três condições: (1) a ingestão enecessidades denutrientes devem ser independentes, (2) a distribuição das necessidades deve ser simétrica em torno da média, o EAR, e
(3) a variância da distribuição das necessidades deve ser menor que a variância da distribuição da ingestão usual $21,35,44$.

Para a vitamina $C$, essas pressuposições são satisfeitas, o que permitiu a utilização do ponto de corte para esse nutriente. Entretanto, para o ferro a simetria da distribuição denecessidadenão ocorre e o seu EAR foi derivado do modelo fatorial. N esses casos, as estimativas de prevalência baseadas na abordagem do ponto de corte fornecem medidas tendenciosas, justificando a indicação do uso da abordagem probabilística ${ }^{35,42}$. 0 modelo fatorial considera a perda basal de ferro, perdas menstruais, necessidades fetais na gestação, necessidades aumentadas durante 0 crescimento ou aumento tecidual, além dos estoques, determinando que mulheres pós-menarca necessitam de maiores quantidades desse mineral ${ }^{45}$.

Com relação à ingestão dos minerais, foi utilizado o EAR de 7,9mg/dia para ferro e de $56 \mathrm{mg} /$ dia para a vitamina $C$, considerando o estágio de vida de catorze a dezoito anos de idade para mulheres. N esse sentido, observa-se que, apesar da média de ingestão em cada fase do ciclo e a média total, quando se considerou a média de seis registros ter sido superior àEAR, encontrouse uma prevalência de inadequação na população de $22,5 \%$ para o ferro e $34 \%$ para a vitamina C. Diferentemente, ao se comparar a média de ingestão à do $\mathrm{N}$ ational Research Council ${ }^{37}$, obteve-se $63,3 \%$ de inadequação para o ferro e $20 \%$ de adequação para a vitamina C, o que evidencia a grande diferença entre os resultados da utilização dos dois métodos, principalmente com relação ao ferro.

Nesse sentido, devemos considerar que, apesar da utilização da Recommended Dietary Allowance ${ }^{37}$ para a avaliação da adequação de dietas, as DRI publicadas desde 1997 substituem o valor único do $\mathrm{N}$ ational Research Council ${ }^{37}$ por quatro categorias devalores de referência - EAR, RDA, AI, UL - para avaliação e planejamento de consumo de indivíduos e coletividades. Dessa forma, ao utilizar-se o EAR, considera-se um valor médio de ingestão diária que atenda às necessidades de $50 \%$ dos indivíduos saudáveis de um mesmo grupo em determinado estágio de vida e gênero. Isso é relevante, uma vez que não seconhece a necessidade verdadeira de indivíduos, mesmo que esses façam parte de um mesmo grupo, diferentemente das pressuposições do National Research Council de 1989. Além disso, as DRI norteiam pela redução do risco de doenças por deficiência ou excesso denutrientes, juntamente com outros parâmetros de saúde. 
Estudos realizados com adolescentes no município deSão Paulo para avaliação deinadequação deferro pela porcentagem do $N$ ational Rese arch Council ${ }^{37}$ encontraram $64 \%$ em Urbano et al. ${ }^{18}$ e $83 \%$ em Garcia et al. ${ }^{38} ; 11,3 \%{ }^{46}$ e $19 \%$ em Albano ${ }^{34}$. A ingestão de ferro para esse mesmo grupo também têm sido colocada entre $11,31^{46} \mathrm{e}$ $16,1 \mathrm{mg} / \mathrm{dia}^{47}$. Em outra população, a média de ingestão de ferro para todas as mulheres no Estados Unidos foi de $12,8 \mathrm{mg}$ em $1988-94$ e $13,4 \mathrm{mg}$ em 1999-2000. Para mulheres, a média de ingestão em ambos os períodos de tempo foi maior do que a EAR para o ferro. Entretanto, as taxas de anemia durante o mesmo período de tempo foram de 2 a $5 \%{ }^{3}$.

Dados de al guns países selecionados sobre 0 percentual de mulheres de 15 a 49 anos mostram que o Brasil possui $21 \%$ de mulheres com anemia por deficiência de ferro, enquanto Angola e Bolívia possuem 59 e 30\%, respectivamente. Em cada um desses países, os fatores significantes que causam a anemia são ingestões baixas em ferro e dietas com ferro de pouca biodisponibilidade. Infelizmente, em várias décadas, desde 0 início das mensurações da deficiência de ferro, pouco progresso foi feito para o combate desse problema, que afeta atétrês quartos de mulheres gestantes, o qual sempre foi visto como um "problema de mulher" 3 .

A vitamina $C$, além de contribuir para a absorção do ferro, age fisiologicamente como antioxidante hidrossolúvel, em virtude de seu alto poder redutor. É também cofator de enzimas envolvidas na biossíntese do colágeno, carnitina e neurotransmissoresin vitro, epodeextinguir uma variedadede espécies reativas deoxigênio enitrogênio no meio aquoso. Santos et al. ${ }^{48}$, trabalhando com questionário defrequência alimentar qualitativo para avaliar o consumo al imentar deadolescentes, observaram baixa frequência no consumo de frutas, fonte importante de vitamina $C$, o que compromete a biodisponibilidade de ferro. Já Albano e Souza ${ }^{34}$ encontraram uma adequação de vitamina C de $318 \%$, associado a grande consumo de frutas pelos adolescentes estudados.

No presente trabalho, observou-se que as adolescentes apresentaram inadequação de $20 \%$ para o ferro, a qual não foi favorecida pelo aumento da biodisponibilidade causada pelo maior consumo de vitamina $C$, uma vez que a inadequação dessa vitamina foi ainda maior. Pode-se associar esses resultados à prática alimentar desajustada, com ausência de refeições, troca dessas por outras mais rápidas, compostas por pão, acompanhamento ebebida, o que comprometeu tanto o consumo do ferro quanto da vitamina $C$.

Esses resultados demonstram que 0 padrão alimentar qualitativo necessita de ajustes para prevenir deficiências nutricionais específicas que podem repercutir na saúde e estatura final. Uma possível explicação para essa questão decorre da contestação do padrão alimentar da família, comportamento esperado na adolescência $a^{45,49}$. Asinadequações observadas em ambos os nutrientes possivelmenterefletem a possibilidadedecomprometimento da saúde futura desses indivíduos ${ }^{11}$.

\section{Conclusão}

A avaliação do consumo de alimentos é importante como indicador indireto do estado nutricional. N esse sentido, objetivou-se estudar a prevalência de inadequação nutricional do ferro evitamina $\mathrm{C}$ em adolescentes pós-menarca, utilizando-se como referência a DRI-EAR e o National Research Council ${ }^{37}$, evidenciando o método manual deanálise deinadequação do ferro, pela sua simplicidade derealização. Encontrou-segrande divergência entre os resultados pelos dois métodos, como inadequação deferro de $22,6 \%$ e63,3\% e vitamina C de $34 \%$ e $20 \%$, para as DRI-EAR e $\mathrm{N}$ ational Research Council ${ }^{37}$, respectivamente. A pesar dessa divergência, ambos os resultados apontam um risco nutricional para a população estudada, considerado um grupo extremamente vulnerável. N esse sentido, sugere-se a realização de estudos dietéticos mais amplos, utilizando as novas recomendações, favorecendo diagnósticos nutricionais com subsequente avaliação de medidas de intervenção, o que poderá contribuir para a prevenção do risco de desenvolvimento de patologias e melhoria da qualidade de vida desse grupo populacional. 


\section{Colaboradores}

L Azevedo trabalhou na concepção e delineamento da pesquisa, avaliação dos resultados, redação e revisão crítica. HSD M artino trabalhou na discussão, análise dos resultados e revisão crítica. FG Carvalho realizou o levantamento de dados, análise estatística e redação do artigo. M L Rezende trabal hou na análise estatística e avaliação dos resultados.

\section{Referências}

1. World Health Organization. Nutrition in adolescenceissues and challenges for the health sector: issues in adolescent health and development. Geneva: WHO; 2005.

2. Vitolo MR. Nutrição da gestação à adolescência. $1^{\text {a }}$ ed. Rio de Janeiro: Reichmann \& Affonso Editores; 2003.

3. Kennedy E, M eyers L. Dietary Reference Intakes: development and uses for assessment of micronutrient status of women- a global perspective. Am J Clin Nutr 2005; 81(Suppl):1194S-1197S.

4. Akabas SR, Dollins KR. M icronutrient requirements of physical active women: what can we learn from iron? Am J Clin N utr 2005; 81(suppl):1246S-1251S.

5. Bertolin MNT. Estágios da mudança de comportamento e sua relação com o consumo alimentar de adolescentes [dissertação]. São Paulo (SP): Faculdade de Saúde Pública, Universidade de São Paulo; 2006.

6. Terres NG, Pinheiro RT, Horta BL, Pinheiro KAT, Horta LL. Prevalência e fatores associados ao sobrepeso e à obesidade em adolescentes. Rev. Saude Publica 2006; 40(4):627-633.

7. Souza EL, Pinto ICS, Lima M A, Targino DM G. Parâmetros nutricionais de dietas de emagrecimento, disponíveis em revistas não científicas impressas. Hig. Aliment 2006; 20(139):27-33.

8. Tarasuk V, Beaton GH. M enstrual-cycle patterns in energy and macronutrient intake. Am J Clin Nutr 1991; 53:442-447.

9. Abalkhail B, Shawky S. Prevalence of daily breakfast intake, iron deficiency anemia and awareness of being anemic among Saudi school students. International Journal of Food Sciences \& N utrition 2002; 53(6):519-530.

10. Assunção MAF, Santos IS. Efeito da fortificação de alimentos com ferro sobre anemia em crianças: um estudo de revisão. Cad Saude Publica 2007; 23(2):269281.

11. Godoy FC, Andrade SC, M orimoto JM, Carandina L, Goldbaum M, Barros M BA, Cesar CLG, Fisberg RM . Índice de qualidade da dieta de adolescentes residentes no distrito do Butantã, município de São Paulo, Brasil. Rev. Nutr. 2006; 19(6):663-671.

12. Cook JD, Reddy MB. Effect of ascorbic acid intake on nonheme-iron absorption from a complete diet. Am J of Clin Nutr 2001; 73(1):93-98.

13. Vitolo MR, Bortolini GA. Iron bioavailability as a protective factor against anemia among children aged 12 to 16 months. J. Pediatr. (Rio J.) 2007; 83(1):33-38.

14. Reddy, MB, Hurrel RF, Cook JD. Meat consumption in a varied diet marginally influences nonheme iron absorption in normal individuals. J Nutr. 2006; 136(3):576-581.

15. Chernoff R. Micronutrient requirements in older women. Am J Clin Nutr 2005; 81(suppl):1240-1245.

16. Bianculli $\mathrm{CH}$. Crescimento físico y endocrinologia en la pubertad. In: Organización Panamericana de La Salud. La salud del adolescente y del joven. Washington, D.C.: OPAS; 1995. p. 87-94.

17. Azevedo L, Oliveira FLR, Martino HSD, Freire GER. Estudo do consumo alimentar e composição corporal de adolescentes durante as fases do ciclo menstrual. Belo Horizonte: FAPEM IG; 2006. [Relatório de pesquisa] 
18. Urbano MDR, Vitalle MSS, Juliano Y, Amâncio OMS. Ferro, cobre e zinco em adolescentes no estirão pubertário. J. Ped. (Rio J.) 2002; 78(4):327-334.

19. Williams S, Dickson N. Early growth, menarche, and adiposity rebound. Lancet 2002; 359(1):580-581.

20. Institute of Medicine. National Academy of Sciences. Dietary reference intakes for vitamin $C$, vitamin $E$, selenium, and carotenoids. Washington, D.C.: National Academy Press; 2000.

21. Slater B, Marchioni DL, Fisberg RM. Estimando a prevalência da ingestão inadequada de nutrientes. Rev. Saude Publica 2004; 38(4):599-605.

22. Fisberg RM, Slater B, Barros RR, Lima FD, Carandina L, Barros M BA, Goldbaum M. Índice de qualidade da dieta: avaliação e aplicabilidade. Rev. Nutr. 2004; 17(3):301-318.

23. Saint M artin B, Slencio, JL, Bourges H. Gómez E, Díaz Sánchez $V$, Boeck $L$. Variación en la ingestión de energía y sus Fuentes durante el ciclo menstrual. Perinatol. Reprod. Hum 1993;7(1):2-7.

24. Sampaio HAC. Aspectos Nutricionais relacionados ao ciclo menstrual. Rev. Nutr. 2002; 15(3):309-317.

25. Giachetto SM P, Solange, M P, Godoy, M F, Almeida I. Comportamento do teste ergométrico em relação as fases do ciclo menstrual. Arq. Ciênc. Saúde 2004;11(2):109-112.

26. Costa COM, Santos CAST, Nascimento Sobrinho C, Moura M SQ, Souza KEP, Assis DR. Gravidez na adolescência: associação de variáveis sociodemográficas e biomédicas materna com resultado neonatal. Rev baiana saúde pública 2005; 29(2):300-312

27. Oliveira MAA, Osório M M, Raposo M CF. Socioeconomic and dietary risk factors for anemia in children aged 6 to 59 months. J. Pediatr. (Rio J.) 2007; 83(1):39-46.

28. Sales RL, Santana M M, Costa NMB. Avaliando 0 consumo alimentar por fotos. [CD-ROM ]. Viçosa: UFV; 2004.

29. Fisberg RM, Slater $B, M$ archioni DM L, M artini LA. Inquéritos alimentares: métodos e bases científicos. Barueri: Manole; 2005.

30. M onteiro JBR, Esteves EA. Diet Pro, versão 4.0: Sistema de suporte à avaliação nutricional e prescrição de dietas [CD-ROM ]. Viçosa: Agromídia Software; 2001.

31. Instituto Brasileiro de Geografia e Estatística. Estudo Nacional de Despesa Familiar: Tabela de composição de alimentos. Rio de Janeiro: IBGE; 1997.

32. Franco G. Tabela de composição química dos alimentos. 9a ed. São Paulo: Atheneu; 2004.

33. Pinheiro ABV, LacerdaEMA, Benzecry EH, Gomes M CS, Costa VM. Tabela para avaliação de consumo alimentar em medidas caseiras. 5a ed. São Paulo: Atheneu; 2004.

34. Albano RD, Souza SB. Ingestão de energia e nutrientes por adolescentes de uma escola pública. J. Pediatr. (Rio J.) 2001; 77(6):512-516.

35. Institute of M edicine. National Academy of Sciences. Dietary reference intakes for vitamin A, vita$\min \mathrm{K}$, arsenic, boron, chromium, copper, iodine, iron, manganese, molybdenum, nickel, silicon, vanadium, and zinc. Washington, D.C.: National Academy Press; 2001. p. 65-126.
36. Jekel JF, Elmore JG, Katz DL. Epidemiologia, bioestatística e medicina preventiva. Porto Alegre: Artmed; 1999.

37. National Research Council. Commission of Life Sciences. Food and Nutrition Board. Recommended Dietary Allowances. $10^{\text {th }}$ ed. Washington, D.C.: National Academy Press; 1989.

38. Garcia GCB, Gambardella AMD, Frutuoso MFP. Estado nutricional e consumo alimentar de adolescentes de um centro de juventude da cidade de São Paulo. Rev. Nutr. 2003; 16(1):41-50.

39. Frankovich RJ, Lebrun CM. The athletic woman: menstrual cycle, contraception, and performance. Clinics in Sports M edicine 2000; 19(2):251-271.

40. Junqueira LC, Carneiro J. Histologia básica. 8a ed. Rio de Janeiro: Guanabara Koogan; 1995.

41. M antoanelli G, Vitalle MSS, Amâncio OMS. Amenorréia e osteoporose em adolescentes atletas. Rev. Nutr. 2002; 15(3):319-340.

42. Padovani RM, Amaya-Farfáni J, Colugnati FAB, Domene SM A. Dietary reference intake: aplicabilidade das tabelas em estudos nutricionais. Rev. Nutr. 2006; 19(6):741-760.

43. Carriquiry AL. Assessing the prevalence of nutrient inadequacy. Public Health Nutrition 1998; 2(1):23-33.

44. Marchioni DML, Slater B, Fisberg RM. A plicação das Dietary References Intake na avaliação da ingestão de nutrientes para indivíduos. Rev. Nutr. 2004; 17(2):207-216.

45. Priore SE. Composição corporal e hábitos alimentares de adolescentes: uma contribuição à interpretação de indicadores do estado nutricional [tese]. São Paulo (SP): Escola Paulista de Medicina, Universidade Federal de São Paulo; 1998.

46. Albano RB. Estado nutricional e consumo alimentar de adolescentes [dissertação]. São Paulo (SP): Faculdade de Saúde Pública, Universidade de São Paulo; 2000.

47. Sichieri R. Epidemiologia da obesidade. Rio de Janeiro: UERJ; 1998.

48. Santos JS, Costa M CO, Nascimento Sobrinho CL, Silva MCM, Souza KEMP, M elo BO. Perfil antropométrico e consumo alimentar de adolescentes de Teixeira de Freitas - Bahia. Rev. Nutr. 2005; 18(5):623-632.

49. Gambardella AM D, Frutuoso MFP, Franch C. Prática alimentar de adolescentes. Rev. Nutr. 1999; 12(1):5-19.

Artigo apresentado em 11/09/2007

Aprovado em 15/07/2008

Versão final apresentada em 06/08/2008 